\title{
Extremum Seeking Control for Beam Steering using Hypersurfaces
}

\author{
Nouman Ashraf, Marios Lestas, \\ Taqwa Saeed \\ Dep. of Electrical Engineering, \\ Frederick University. \\ Dep. of Computer Science, \\ University of Cyprus. \\ Nicosia, Cyprus. \\ nouman.ashraf@stud.frederick.ac.cy
}

\author{
Hamidreza Taghvaee, Sergi Abadal \\ Dep. of Computer Architecture, \\ Universitat Politècnica de Catalunya. \\ Barcelona, Spain. \\ abadal@ac.upc.edu
}

Andreas Pitsillides

Dep. of Computer Science,

University of Cyprus.

Nicosia, Cyprus.

andreas.pitsillides@cs.ucy.ac.cy

\author{
Christos Liaskos \\ Institute of Computer Science, \\ Foundation of Research and Technology \\ Hellas. \\ Heraklion, Greece. \\ cliaskos@ics.forth.gr
}

\begin{abstract}
Hypersurface, a software defined metasurface (SDM) paradigm constitutes a revolutionary technology aiming at offering programmatic control over all aspects of a propagating wave, altering its direction, power, polarization and phase. In the absence of line of sight (LOS) between a transmitter and a receiver, HSF can provide seamless connectivity via programmatic reflection. However, such an application requires fine tuning of the metasurface reconfiguration parameters, which may not be effective when done in an open loop fashion due to model uncertainties. In this work, we consider a feedback-based formulation of the problem, which involves maximization of the received power and propose the use of Extremum Seeking Control (ESC) for the controller implementation due to fact that it is model-free and adheres to the maximization problem. Extensive simulations indicate that the proposed scheme is successful in guiding the impinging wave to the receiver within reasonable time even in the presence of time varying delays incurred by message propagation. In addition, a discrete time implementation is considered, investigating its limitations as we increase the sampling time while also characterizing the traffic within the controller network.

Index Terms-Beam steering, extremum seeking control, mm-wave and terahertz communication, intelligent metasurface, HyperSurface.
\end{abstract}

\section{INTRODUCTION}

Recently, Software-defined Metasurfaces (SDMs) have received considerable attention, due to their capability to fully control electromagnetic (EM) waves during their propagation from a transmitter to a receiver [1]. In particular, SDMs allow for total and precise control over the direction, polarization, amplitude and phase of impinging waves, in a frequency and encoding-selective manner [2]. These exquisite capabilities have recently enabled the Programmable Wireless Environment (PWE) concept [3], i.e., SDM-coated spaces such as floorplans, within which the propagation of Terahertz (THz) waves emitted from communication devices becomes completely software-defined, mitigating the loss of seamless connectivity phenomena in non-line-of-sight (NLOS) THz communication [4].

This work was funded by the European Union via the Horizon 2020: Future Emerging Topics call (FETOPEN), grant EU736876, project VISORSURF (http://www.visorsurf.eu).
From a physical standpoint, SDMs comprise sub-wavelength antennas, each typically smaller than $\lambda / 10, \lambda$ being the minimal wavelength of the impinging waves to be controlled. These miniaturized antennas are periodically repeated in 2-D layouts or even 3-D stackups, hence resembling overall a material composed of a meta-atom (i.e., the periodically repeated element) rather than an antenna array or a reflectarray [5]. State-altering elements are regularly intertwined within the meta-atoms, allowing for a timevariant and programmable interaction between the SDM and the impinging waves. Recently, the idea of implementing SDMs with an embedded controller network for disseminating packetized directives, to set the meta-atoms, together with specialized software and a well-defined application programming interface (API) has emerged, leading to HyperSurfaces (HSFs) [6].

It is noted that for operation in the $\mathrm{THz}$ regime, the corresponding physical implementation diverts from the described convention of $\lambda / 10$-sized meta-atoms. Instead, it relies on a capacitative structure comprising a Graphene sheet and a patterned metallic layer, with a non-linear, tunable dielectric layer (e.g. ion-gel) in-between. The tunable dielectric is biased to match the impinging wave impedance, while the patterned metallic layer is piece-wise biased by state-altering elements to control the local phase shift across the surface [7].

One of the biggest challenges in the deployment of fifth generation (5G) and beyond will be the need for maintaining NLOS connection among nodes, especially in the context of the directional $\mathrm{THz}$ and $\mathrm{mm}$-wave frequency band. These highly directional $\mathrm{THz}$ communications undergo blockage because of obstacles such as high rise buildings and walls in outdoor urban and indoor environments respectively, thereby highlighting the need for a solution to sustain seamless connectivity. Beam steering via reflection on an HSF can serve this purpose [4].

As pointed out in literature [8], metasurfaces have distinct advantages in realizing beam steering applications relative to competing technologies as for example backscatter communication, multi-input multi-output (MIMO) beamforming and relaying: passive nature, capable of supporting soft 


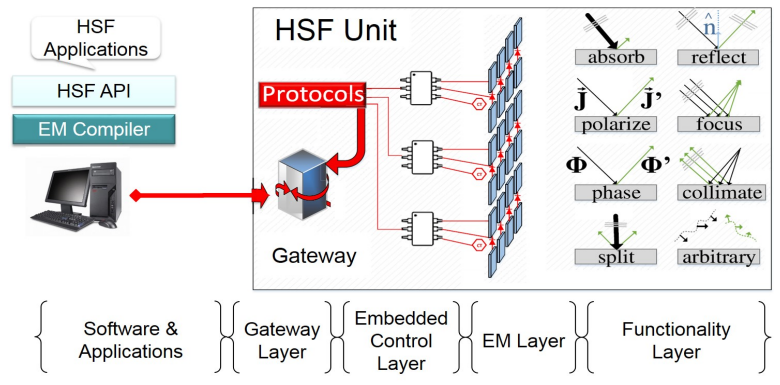

Fig. 1: Layers of HSF system

programming, resilient to noise, as metasurfaces do not need digital-to-analog (DA) and analog-to-digital (AD) converters, and power amplifiers (PAs), full-band operating frequency and easy deployment (e.g. on the ceilings of factories and indoor spaces, on the facades of buildings, human clothing, etc.).

In this work, we consider the problem of establishing reliable communication between a transmitter and a receiver via an intelligent reconfigurable HSF, by appropriately tuning its states. Assuming uncertainties in the underlying model (e.g., stemming from hardware imprecision or localization error margins), we consider a closed loop scheme and formulate the problem mathematically aiming to maximize the power at the receiver. In our previous work [9], we have considered a certainty equivalent controller based on extended Kalman filtering (EKF) for state estimation using the number of unit cells as the control variable. Use of feedback control for metamaterial aided beam steering has also been reported in the very recent paper [10] published in Nature Light. However, recent progress has enabled us to consider the reflection angle as the control variable which we couple with Extremum Seeking Control (ESC). ESC has been preferred due to its model free nature and the fact that, inline with the problem objective, it aims at maximising the control output. The proposed scheme is shown through a number of simulations to converge to the desired state in reasonable time even in the presence of time varying delays. The considered feedback delays mainly stem from the time it takes for the packetized directives to be disseminated to the controller nodes within the embedded controller network (CN). These are obtained using a custom developed simulator based on the Anylogic Tool [11]. In addition, a discrete time implementation of the algorithm is considered and the effect of the sampling time is assessed on both the controller performance and on the traffic generated within the controller network.

\section{HyPERSURFACE SYSTEM DESCRIPTION}

\section{A. Hypersurface Structure}

Fig. 1 depicts the HSF system's physical and functional architecture, consisting of mainly the EM layer, the functionality layer, the embedded control layer and the gateway layer.

The functionality layer includes EM control that can be applied to the impinging beam, either separately or in combination with the corresponding application programming interface (API) calls. The EM layer actually realizes the EM functionalities and can be composed of copper [12] or even graphene patches [13], the metaatom, arranged periodically on a silicon substrate terminated by a metal ground plane. When the EM layer is impinged by a plane wave, it induces local currents in the patches that function as secondary EM sources modifying the scattered field to realize the desired functionality. Note that such a reaction is macroscopically defined by the real and imaginary surface impedance of the EM layer and, thus, we need to dynamically adjust such values to modify the metasurface response. To this end, let us assume that two tunable lumped components, $R$ (varistor) and $C$ (varactor), are integrated behind the back-plate to exert control over the real and imaginary surface impedance, respectively.

An embedded control layer [12] exists behind the EM layer, which includes the hardware and protocols required for transferring information between the gateway (GW) and the state alteration components and to actually change the state of the meta-atoms. The control layer, in its simplest form, can be the direct cabling of the state changing elements to the GW that can be sufficient for even some PWE applications [14]. However, with the increase in density of meta-atoms, a network of embedded data controllers, referred to as $\mathrm{CN}$ in this paper, is needed to exert control within the HSF. It is generally assumed that each controller can only apply $N_{s}$ states, which represent $N_{s}$ combinations of $R C$ values.

Behind the embedded control layer is the GW layer, which (i) provides a connection of HSF to the external world via standardized protocols and (ii) turns software directives into particular metasurface codes. The metasurface code is the set of meta-atom states ( $R C$ values, out of the $N_{s}$ available states) at which the array of meta-atoms of the HSF has to be established to achieve the desired functionality. To apply this code, the GW sends a series of simple internal control messages that trigger the appropriate state changes to the different meta-atoms.

\section{B. Metasurface Coding}

In this subsection, we discuss how the metasuface code is determined for the particular case of anomalous reflection for beam steering. As pointed out in [15], anomalous reflection can be achieved through the implementation of phase gradients across the HSF. In other words, the $R C$ values of each meta-atom need to be tuned to obtain a reflection phase with linear gradients $\Phi_{x}^{\prime}$ and $\Phi_{y}^{\prime}$ in the $x$ and $y$ directions, respectively. As a result, the phase of a meta-atom at location $(i, j)$, i.e. the $i$-th column and $j$-th row, can be expressed as:

$$
\Phi_{i j}=\left(\Phi_{x}^{\prime} i+\Phi_{y}^{\prime} j\right) D_{u}
$$

where $D_{u}$ is the lateral size of the meta-atom. Since the number of meta-atom states is limited to $N_{s}$, the actual phase is mapped to that of the closest state [16]. In order to relate the target reflected angle $\left(\left\{\theta_{r}, \phi_{r}\right\}\right.$ in polar coordinates) with the angle of incidence $\left(\left\{\theta_{i}, \phi_{i}\right\}\right)$ and the phase gradients implemented in the HSF $\left(\Phi_{x}^{\prime}\right.$ and $\left.\Phi_{y}^{\prime}\right)$, the momentum conservation law for wave vectors can be applied, leading to:

$$
\begin{aligned}
& \Phi_{x}^{\prime}=k_{r} \sin \theta_{r} \cos \phi_{r}-k_{i} \sin \theta_{i} \cos \phi_{i} \\
& \Phi_{y}^{\prime}=k_{r} \sin \theta_{r} \sin \phi_{r}-k_{i} \sin \theta_{i} \sin \phi_{i},
\end{aligned}
$$

where $k_{i}=\frac{2 \pi}{\lambda_{i}}$ and $k_{r}=\frac{2 \pi}{\lambda_{r}}$ are the wave vectors of the incident and reflected mediums with wavelengths $\lambda_{i}$ and $\lambda_{r}$, respectively. Whenever the incidence or reflected angle change, the HSF coding will need to change as well to accommodate the new required phase gradients. 


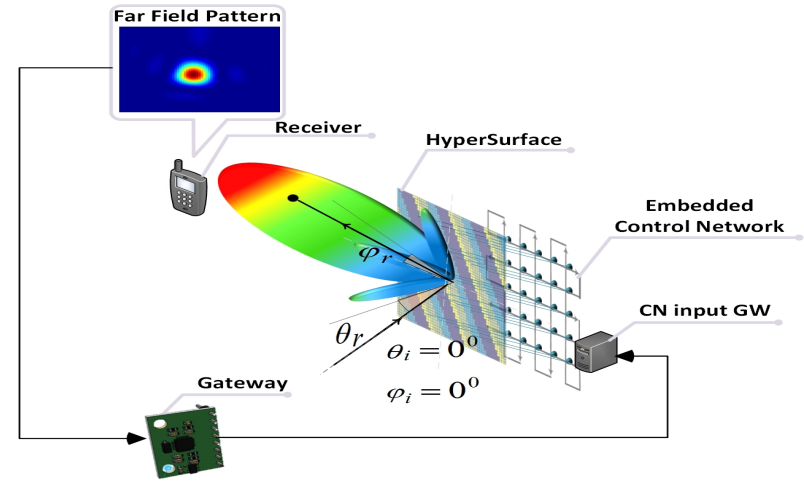

Fig. 2: Physical layout of the system

\section{Far Field Evaluation}

This subsection describes how the Far Field pattern is evaluated as a function of the meta-atom states. Such a model is necessary to determine the signal power at the receiver, which is related to the value of radiation pattern of the reflected beam at the receiver location. This value is affected by the beamwidth and side lobes that appear in the reflected beam due to the finite aperture of the HSF.

To evaluate the Far Field pattern of a metasurface, we apply the Huygens principle by which the far field is the sum of the contributions of all meta-atoms. This model assumes that the crosstalk between adjacent meta-atoms can be neglected. We further assume that meta-atoms are isotropic and that the reflection amplitude is constant across all the states. With these assumptions, and for linearly polarized normal incidence and assuming full metasurface illumination, the scattering field can be expressed as:

$$
E(\theta, \phi)=K \sum_{i=1}^{M} \sum_{j=1}^{N} e^{j\left[\Phi_{i j}+k_{0} \zeta_{i j}(\theta, \phi)\right]},
$$

where $K$ is the reflection amplitude constant, $\Phi_{i j}$ is the reflection phase of meta-atom $(i, j), M$ and $N$ are the number of unit cells in a row or a column, $k_{0}$ is the wave number and $\zeta_{i j}(\theta, \phi)$ is the relative phase shift of the unit cells with respect to the radiation pattern coordinates $(\phi, \theta)$,

$$
\zeta_{i j}(\theta, \phi)=D_{u} \sin \theta\left[\left(i-\frac{1}{2}\right) \cos \phi+\left(j-\frac{1}{2}\right) \sin \phi\right] .
$$

This method has proven to be accurate in evaluating the far field of a metasurface for beam steering by comparing the results with those of full-wave simulations [17]. The approximations made have a small impact on the value and position of the side lobes, which are of minor relevance to the purpose of this work.

\section{BeAm SteEring Algorithm}

\section{A. Problem Formulation}

The considered scenario involves a transmitter and receiver establishing a reliable communication channel via the HSF, due to the absence of LOS capability. The objective is to tune the configuration state of the reconfigurable metasurface so that optimal channel quality is achieved. In this work, the channel quality is assessed by the received power at the receiver, which is aimed to be maximized. We consider the scenario depicted in Fig.
2, where a single beam (emanating from a fixed transmitter) is incident on the metasurface with a fixed angle of incidence. The angle comprises of 2 components with $\theta_{i}$ denoting the elevation angle and $\phi_{i}$ denoting the azimuth angle. Without loss of generality, we assume the incidence angles in the conducted experiments to be equal to 0 . The beam is reflected on the metasurface with the angle of reflection comprising of the elevation and azimuth components denoted by $\theta_{r}(t)$ and $\phi_{r}(t)$ respectively. Due to the unique characteristics of the electromagnetic behaviour of the metasurface, the energy of the reflected wave is not fully focused, leading to a sidelobe pattern characterizing the Far Field pattern. A receiver is present at specific location $l$, in $3 \mathrm{D}$ space which measures the received power $P(t)$. The metasurafce, as discussed in the previous section comprises of a finite set of $N$ controllers interconnected via the embedded CN. Each controller assumes one of $L$ finite discrete states. The controller states $c_{1}, c_{2}, \ldots c_{N}$ are lumped into a vector $c=\left[c_{1}, c_{2}, \ldots c_{N}\right]$ with each entry assuming a value from the set $S=s_{1}, s_{2}, s_{3}, \ldots s_{L}$. The incidence and reflection angles are also lumped into the vectors $\psi_{i}=\left[\theta_{i}, \phi_{i}\right], \psi_{r}=\left[\theta_{r}, \phi_{r}\right]$ respectively. The reflection angle $\psi_{r}$ depends on the incidence angle and the controller states such that $\psi_{r}=f\left(c, \psi_{i}\right)$. The function $f($.$) has been obtained in$ tabular form and is highly nonlinear. The chosen controller states $c$, yield a particular Far Field pattern, which results in the received power $P(t)$. The latter is thus also a function of the controller states $c$, the incidence angle $\psi_{i}$ and the location $l$ of the receiver such that $P(t)=g\left(c, \psi_{i}, l\right)$. The problem is thus to choose the controller states such that the received power is maximized, i.e, $\max _{c \in C} P(t)$. However, the multidimensionality of the input space and the highly nonlinear and uncertain nature of the function $g($.$) renders the problem difficult to solve and we thus reduce$ the complexity by considering a single input variable, namely the reflection angle $\psi_{r}$ whose relationship with the controller states $c$ is described by the function $g($.$) . This change in the$ input space also ensures the concavity of $P(t)$ with respect to the input variable $\psi_{r}$, which is important in the application of ESC. So the considered optimization problem becomes:

$$
P: \max _{\psi_{r} \in[-90,90]} P(t) \text {. }
$$

The aforementioned problem faces a number of challenges.

- The highly nonlinear nature of the functions $f($.$) and g($.$) and$ the uncertainty in their specification due to the inaccuracies at the construction phase and the possibility of faulty behaviour of both the metasurface and the $\mathrm{CN}$.

- The presence of disturbances, as for example, background noise at the received power measurements.

- The single point of measurement in $3 \mathrm{D}$ space at the location of the receiver.

To address these challenges, and especially the robustness issue with respect to uncertainties, a closed loop implementation has been preferred as opposed to open loop control [18]. For closed loop implementation, we assume that the power measured at the receiver is communicated to the input gateway of the HSF. The input gateway then processes this measurement according to the control algorithm to be designed and generates the desired reflection angle $\psi_{r}$. The latter is then transformed into the controller states $c$, via the inverse of the function $f($.$) , assumed$ to be one-to-one. The desired controller states are communicated 


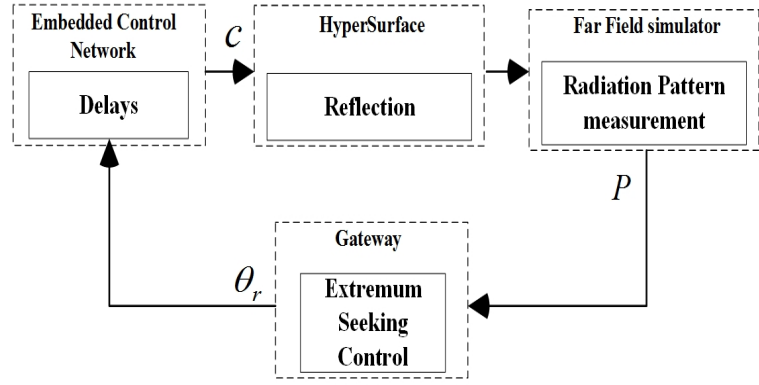

Fig. 3: Block diagram of the closed loop feedback system

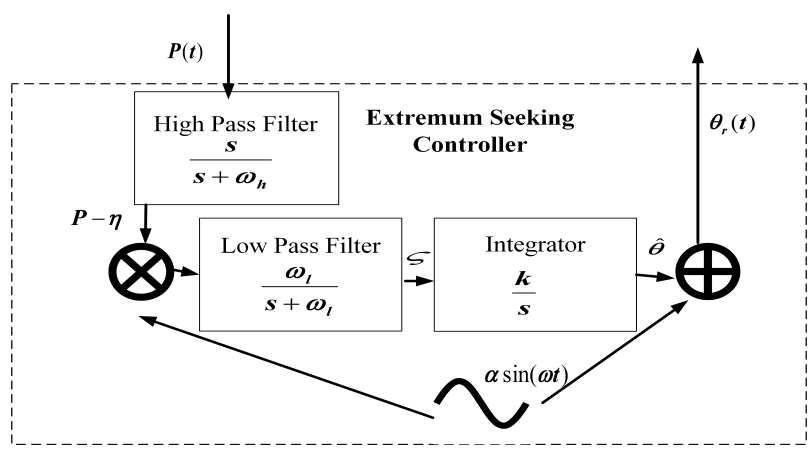

Fig. 4: Proposed ESC for beam steering

to the corresponding controllers via the embedded controller network. This communication is an active area of research and has been addressed in our recent works in [19]. Here, we assume the XY algorithm, which works effectively in the absence of faults and consider unicast routing one-by-one to the corresponding controllers. This information dissemination process incurs some delay $d(t)$ and might compromise the stability of the system, an issue which is investigated further in the performance evaluation section. Once the controller messages are sent, the controller states abide to these directions, the Far Field pattern is modified and the procedure is repeated. Here, we assume a feedback controller of the form:

$$
\left\{\begin{array}{l}
\dot{x}=h(x, P) \\
\psi_{r}=v(x, P),
\end{array}\right.
$$

where $\psi_{r}(t)$ is the desired angle of reflection, $P(t)$ is the measured signal strength, $x$ is a vector of controller states and $h($.$) and v($.$) are possibly nonlinear functions. The problem is$ then to design the functions $h($.$) and v($.$) such that problem P$ is solved. A block diagram of the feedback system, as described above is shown in Fig. 3 .

\section{B. Proposed Control Algorithm}

ESC is an adaptive, non-linear and model-free control method, which has been chosen, due to the highly nonlinear, coupled with a significant degree of uncertainty, model of the HSF behavior. In this strategy, the input signal is perturbed sinusoidally and the resulting change in the output signal is measured to determine and follow a local maximum of a measurable objective function. The subsequent discussion refers to the angle of elevation $\theta_{r}$, but it is also applicable for the azimuth angle $\phi_{r}$. The proposed ESC method for the beam steering application under consideration is shown schematically in Fig. 4. $P(t)$ is the system output and thus the controller input and $\theta_{r}(t)$ is the controller output serving as the control variable. As depicted in the Fig. 4, a sinusoidal dither signal $\alpha \sin (\omega t)$ is added to the best estimate of the input signal $\hat{\theta}$ to get a perturbed input signal $\theta_{r} . \theta_{r}(t)$ is used to configure the HSF system and the resulting $P(t)$ is measured at the receiver. Low frequency components are removed from the measured signal by directing it through a High Pass filter (HPF). The signal after filtration denoted by $P_{h}$ is demodulated by multiplying it with the dither signal $\alpha \sin (\omega t)$. This demodulated signal is then integrated to obtain an updated value of $\hat{\theta}$. The continuous time algorithm of the considered ESC scheme of Fig. 4 is given by:

$$
\left\{\begin{array}{l}
P(t)=f\left(\theta_{r}(t)\right) \\
\theta_{r}(t)=\hat{\theta}(t)+\alpha \sin (\omega t) \\
\dot{\hat{\theta}}(t)=k \zeta(t) \\
\dot{\zeta}(t)=-\omega_{l} \zeta(t)+\omega_{l}(P(t)-\eta(t)) \alpha \sin (\omega t) \\
\dot{\eta}(t)=-\omega_{h} \eta(t)+\omega_{h} P(t),
\end{array}\right.
$$

where $k$ is gain of the gradient based update law, $\omega$ is the perturbation frequency, $\omega_{h}$ is the cut-off frequency of the HPF, $\omega_{l}$ is the cut-off frequency of the Low Pass filter (LPF) and $\zeta$ is the demodulated signal. For the stability analysis of ESC, readers are referred to [20]. What is amenable for a practical implementation at the HSF gateway is a discrete time implementation of the continuous time algorithm presented earlier, which is not shown here due to lack of space.

\section{Performance Evaluation}

In this section, we evaluate the performance of the proposed control scheme investigating its ability to converge to the desired operating point and its ability to maintain good performance in the presence of delays incurred by information dissemination within the $\mathrm{CN}$. The traffic patterns within the $\mathrm{CN}$ for different sampling times are also investigated. The evaluation is conducted using two simulators, one developed on Matlab and one developed using the Anylogic simulation platform. The latter models the process of delivering packetized directives within the HSF embedded CN taking into account its unique characteristics, as for example its asynchronous operation. The Matlab simulator comprises of three main components. The controller implementation, the function mapping the reflection angle generated by the controller to the controller states, and the module which characterizes the Far Field pattern as a result of the chosen controller states. The received signal power is measured by the Far Field radiation pattern. To simplify the basic scenario, reducing the considered parameter dimensionality, we have fixed the azimuth angle to $\phi_{r}=80^{\circ}$ and consider only variations in the reflected elevation angle $\theta_{r}$. Similar observations have been reported when azimuth variations are considered. For this reason, the terms reflected elevation angle and reflection angle are used interchangeably. In addition, throughout the evaluation procedure, we consider received power values normalized by the maximum value such that $0 \leq P(t) \leq 1$. The first set of experiments are conducted assuming zero feedback delays. We consider an initial elevation angle equal to $40^{\circ}$ with the receiver placed at $\theta=50^{\circ}$. The time response 


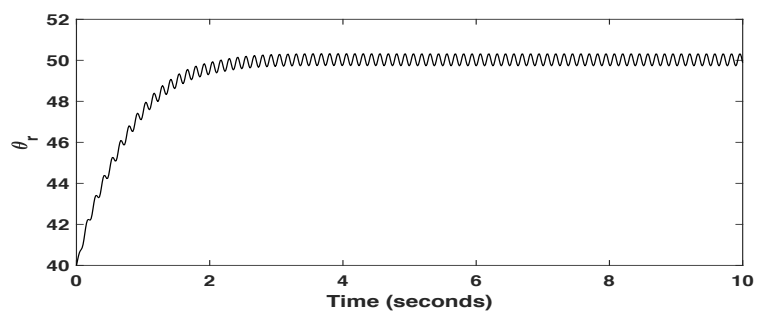

Fig. 5: Time Evolution of the Reflected Elevation Angle

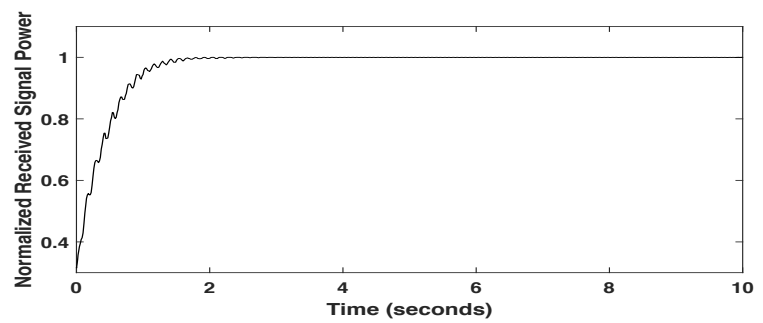

Fig. 6: Time Evolution of the Normalized Received Signal Power measured via the Far Field Simulator

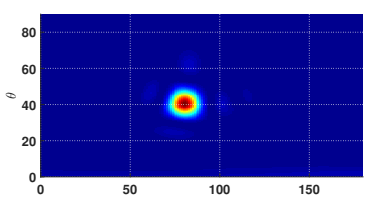

(a) at $t=0 \mathrm{~s}$

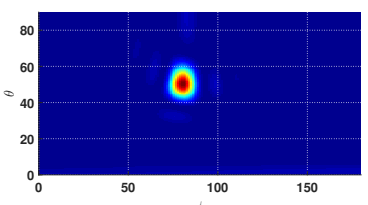

(b) at $t=2.03 \mathrm{~s}$
Fig. 7: Radiation Pattern

of both the reflection elevation angle and the received power are shown in Fig. 5 and 6 respectively. It is demonstrated that the ESC algorithm is able to guide the beam to the correct direction, causing the normalized received power to increase from approximately 0.4 to 1 . The convergence time is, however, rather slow, of the order of a couple of seconds, and a major objective of future research is to further investigate ways with which this convergence time can be reduced, either by trying alternative methodologies or via enhancements of the ESC method. Moreover the radiation pattern at a plane parallel to the HSF which includes the receiver, has been obtained at different simulation instants; at time 0 and at time 2.03 seconds when the system has converged to its steady state response. Their graphical representation as intensity heat maps for different elevation and azimuth angles are shown in Fig. 7a and 7b respectively. We observe that most of the energy is concentrated in a small area about the center point and that the centre is able to shift from $40^{\circ}$ to $50^{\circ}$.

In the next set of experiments, we investigate the effect of delays on the performance of the proposed algorithm. There are 2 sources of delays in the proposed system. There is propagation delay associated with the forwarding of the power measurement from the receiver to the $\mathrm{GW}$, and there is another delay associated with the dissemination of packetized directives from the input $\mathrm{GW}$ to the controller nodes via the embedded $\mathrm{CN}$. We assume that the former is small compared
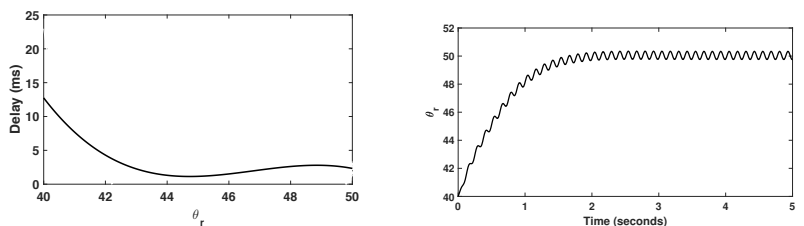

(a) Delay introduced by the embedded (b) Angle of reflection in the case of time control network varying delay

Fig. 8: Time varying delay

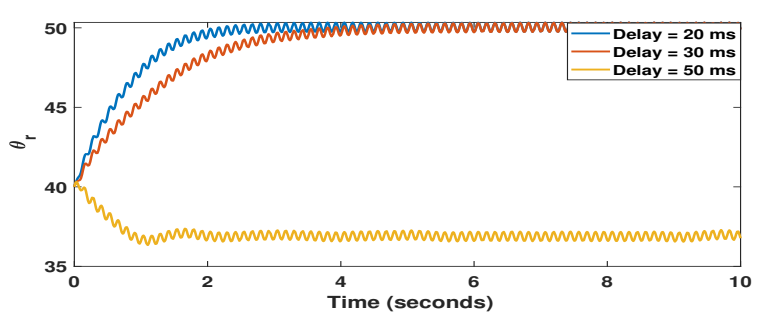

Fig. 9: Angle of reflection with different delays

to the latter and the aim is to characterize the $\mathrm{CN}$ delays and investigate if they are sufficient to render the system to become unstable. The characterization of the $\mathrm{CN}$ delays is done using the Anylogic simulator. As there are currently difficulties in directly interfacing the Matlab and the Anylogic simulators, the delays have been pre-calculated for each reflection angle value. Each reflection angle value corresponds to a controller state configuration. The input GW ensures that the controllers assume these states by sending packetized directives to each one of them. The Anylogic simulator is then used to calculate the total time required for these packetized directives to reach their destination. Simulation results have been conducted to yield $\mathrm{CN}$ delay values for each $\theta_{r}$ and a polynomial fitting procedure has led to the relationship shown in Fig. 8. This relationship when incorporated in the Matlab simulation tool effectively creates a time varying delay which, however, does not lead the system to instability. As shown in Fig. 8(b), convergence to the desired reflection angle is still achieved, with the time evolution becoming slightly more oscillatory.

The question that arises is then the following. What delay values cause the system to behave in an undesirable way? We examine the convergence properties of the system in the presence of feedback delays equal to 20,30 and $50 \mathrm{~ms}$. The resulting time evolution of the elevation angle is shown in Fig. 9. It is observed that at $50 \mathrm{~ms}$ delay the beam is steered to an undesirable direction, which renders the received power equal to 0 . The $50 \mathrm{~ms}$ bound is not very far from the delay values that can be incurred by the $\mathrm{CN}$, so careful consideration of the delay effect is required in future designs.

As discussed in the previous section, the GW will assume a discrete time implementation of the proposed algorithm. In the subsequent analysis, we consider such a discrete time implementation in our simulation framework and investigate the effect of the sampling time $T$ on system performance. We consider sampling time values equal to $0.01,0.05,0.1$ and 0.5 sec. The time evolution of $\theta_{r}$ for each of these sampling times 


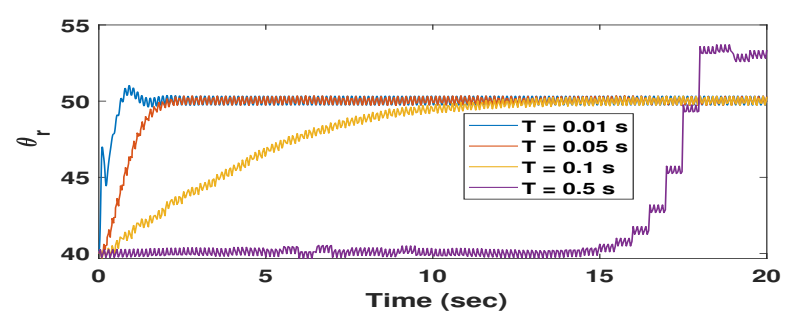

Fig. 10: Angle of reflection for different sampling times

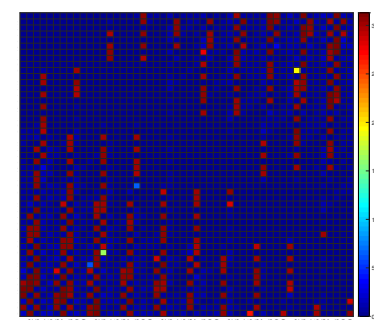

(a) $T=0.01$

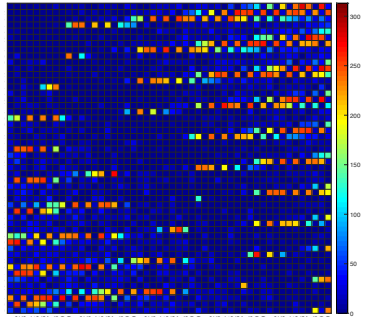

(b) $T=0.5$
Fig. 11: Spatial distribution of traffic generated by algorithm.

is shown in Fig. 10. Increasing sampling times cause an increase in the system damping resulting in slower responses. When $T$ reaches a sufficiently high value $(0.5 \mathrm{sec})$, the system even fails to converge to the desired state, causing the received power to attain values less than 1 .

However, small sampling times implies more frequent update of the controller states, thus, incurring a higher traffic load at the $\mathrm{CN}$. To investigate this effect, we investigate the workload generated by the control algorithm on the HSF CN using the Anylogic simulator. First, we consider the effect of the sampling period on the spatial distribution of traffic in the HSF controller network. We visualize this using heatmaps, the cells of which correspond to the cells of the HSF. The "hotter" the color of the cell the higher is the number of times it has been reconfigured. We assume that the surface is able to detect the slightest change in the reflection angle and reconfigure the unit cells accordingly. It can be observed in Fig. 11 that a small number of cells is frequently configured while a large portion of cells is rarely configured. This can be due to the fact that the changes in the reflection angle are small and thus the phase profile of a small number of cells must be adjusted. As expected, a small sampling period yields a higher number of configuration. Another way with which to assess the traffic load as we increase the sampling time is to investigate the sending rate of packetized directives injected by the gateway. This is depicted in Fig. 12, which shows that as the sampling time decreases the periodic traffic patterns increase in both their frequency and their peak amplitude, until $T$ becomes equal to $0.01 \mathrm{sec}$ where the peak amplitude decreases but the frequency increases even further.

\section{CONCLUSION}

In this paper, we design and evaluate a closed loop scheme for appropriately configuring the HyperSurface (HSF) controller states so that a transmitted beam impinging on the HSF is steered towards a receiver. Due to its model free nature, extremum seeking control (ESC) is chosen for the controller implementation, which is shown through simulations to be effective, even in the

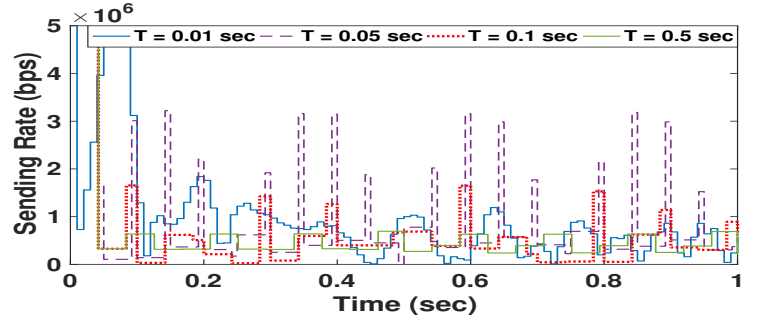

Fig. 12: Sending rate of injected requests versus sampling times

presence of time varying delays emanating from information dissemination latency within the embedded controller network. The traffic within the controller network $(\mathrm{CN})$ is also characterized for different sampling rates. Future work will focus on analytical evaluation of the ESC strategy in the presence of delays, methodologies with which the convergence rate can be increased and consideration of moving targets and multiple impinging waves.

\section{REFERENCES}

[1] C. Liaskos, A. Tsioliaridou, A. Pitsillides, I. F. Akyildiz, and et al, "Design and development of software defined metamaterials for nanonetworks," IEEE Circuits and Systems Magazine, vol. 15, no. 4, pp. 12-25, 2015.

[2] L. Zhang et al., "Space-time-coding digital metasurfaces," Nature communications, vol. 9, no. 1, p. 4334, 2018.

[3] C. Liaskos, A. Tsioliaridou, A. Pitsillides, S. Ioannidis, and I. Akyildiz, "Using any surface to realize a new paradigm for wireless communications," Communications of the ACM, vol. 61, no. 11, pp. 30-33, 2018.

[4] X. Fu et al., "Terahertz beam steering technologies: From phased arrays to field-programmable metasurfaces," Advanced Optical Materials, p. 1900628, 2019.

[5] Q.-U.-A. Nadeem, A. Kammoun, A. Chaaban, M. Debbah, and M.-S. Alouini, "Intelligent reflecting surface assisted multi-user miso communication," arXiv preprint arXiv:1906.02360, 2019.

[6] C. Liaskos, S. Nie, A. Tsioliaridou, et al., "Realizing wireless communication through software-defined hypersurface environments," in IEEE WoWMoM'18, pp. 14-15.

[7] X. Wang and S. A. Tretyakov, "Toward ultimate control of terahertz wave absorption in graphene," IEEE Transactions on Antennas and Propagation, vol. 67, no. 4, pp. 2452-2461, 2018.

[8] E. Basar, M. Di Renzo, J. de Rosny, M. Debbah, M.-S. Alouini, and R. Zhang, "Wireless communications through reconfigurable intelligent surfaces," arXiv preprint arXiv:1906.09490, 2019.

[9] N. Ashraf, T. Saeed, and et al., "Feedback based beam steering for intelligent metasurfaces," in IEEE MENACOMM'19, 2019.

[10] Q. Ma et al., "Smart metasurface with self-adaptively reprogrammable functions," Nature Light: Science \& Applications, vol. 8, no. 1, pp. 1-12, 2019.

[11] The AnyLogic Company, "The anylogic simulator," 2019.

[12] A. C. Tasolamprou et al., "Intercell wireless communication in software-defined metasurfaces," in IEEE ISCAS'18, pp. 1-5.

[13] S. Dash, C. Liaskos, I. F. Akyildiz, and A. Pitsillides, "Wideband perfect absorption polarization insensitive reconfigurable graphene metasurface for thz wireless environment," in IEEE MTTW'19, vol. 1, pp. 93-96.

[14] C. Liaskos, A. Tsioliaridou, S. Nie, A. Pitsillides, S. Ioannidis, and I. F. Akyildiz, "On the network-layer modeling and configuration of programmable wireless environments," IEEE/ACM Transactions on Networking, vol. 27, no. 4, pp. 1696-1713, 2019.

[15] N. Yu, P. Genevet, et al., "Light propagation with phase discontinuities: generalized laws of reflection and refraction," science, American Association for the Advancement of Science, vol. 334, no. 6054, pp. 333-337, 2011.

[16] T. Saeed, S. Abadal, C. Liaskos, A. Pitsillides, H. Taghvaee, A. CabellosAparicio, M. Lestas, and E. Alarcón, "Workload characterization of programmable metasurfaces," in ACM NANOCOM'19, pp. 1-6.

[17] S. E. Hosseininejad et al., "Digital Metasurface Based on Graphene: An Application to Beam Steering in Terahertz Plasmonic Antennas," IEEE Transactions on Nanotechnology, vol. 18, no. 1, pp. 734-746, 2019.

[18] X. Tan et al., "Enabling indoor mobile millimeter-wave networks based on smart reflect-arrays," in IEEE INFOCOM, pp. 270-278, IEEE, 2018.

[19] T. Saeed et al., "Fault adaptive routing in metasurface controller networks," in IEEE Workshop on Network on Chip Architectures, pp. 1-6, 2018.

[20] K. B. Ariyur and M. Krstic, Real-time optimization by extremum-seeking control. John Wiley \& Sons, 2003. 